\title{
Effect of Partial Denervation and Terminal Field Expansion on Neuromuscular Transmitter Release and Nerve Terminal Structure
}

\author{
Sarah Rochel and Norman Robbins \\ Department of Developmental Genetics and Anatomy, Case Western Reserve University, School of Medicine, Cleveland, \\ Ohio 44106
}

\begin{abstract}
The efficacy of evoked ACh release by intact and newly sprouted terminals in response to partial denervation and expansion of the motor neuron terminal field was studied in mouse soleus muscle after section of the L-5 spinal root. From 2 to $4 \mathrm{~d}$ after partial denervation until $90 \mathrm{~d}$ later, only 3-7 motor units of the normal 21 remained. Regeneration of the dissected nerve was prevented while the remaining motor units were sprouting. The indirect twitch, which was only $20 \%$ of direct twitch tension 2-4 d after nerve section, recovered between 28 and $50 \mathrm{~d}$ postoperatively. However, the depression of twitch in low Ca/high Mg solution, which was equal to control 2-3 d postoperatively, was 2-3 times more depressed than control by $\mathbf{5 0} \mathrm{d}$ and remained so up to $90 \mathrm{~d}$. This indicated persistent reduction of the safety factor in sprouted motor units.
\end{abstract}

Intracellular measurement of quantal content in $0.4 \mathrm{~mm} \mathrm{Ca}$, $2.75 \mathrm{~mm} \mathrm{Mg}$ revealed 2 groups of nerve terminals in partially denervated muscle. The quantal content of the first group was greater than contralateral control at earlier times (28$50 \mathrm{~d}$ ) and only slightly greater than control later (74 and 90 d). This group consisted of the original undenervated terminals, since it was associated with normal miniature endplate potential (MEPP) frequency and end-plate potential (EPP) latency, and presumably with the class of fibers with normal (zinc iodide osmium-stained) nerve terminal morphology and occasional large myelinated preterminal axons. The second group had lower quantal content than control at all times, and probably consisted of newly sprouted terminals, since after denervation, MEPP frequency was lower and EPP latency longer than controls. The sprout origin of the second group was consistent with the morphological finding of a population of fibers in which the nerve terminals were smaller in area, showed an increased number of synaptic regions, and were supplied by unmyelinated sprouts.

Thus, when the motor unit size was expanded 3- to 5-fold, ACh release and nerve terminal size were reduced in sprouted terminals, even $\mathbf{9 0} \mathbf{d}$ after nerve section. This reduction may reflect an upper limit for the supply of synaptic components by the perikaryon, with a distribution of components such that a reduced but generally effective safety factor is

\footnotetext{
Received Apr. 25, 1986; revised June 29, 1987; accepted July 7, 1987.

This work was supported by a Fellowship from the National Amyotrophic Lateral Sclerosis Foundation to S.R. and NIH Grants AG00795 and NS18694 to N.R.

Correspondence should be addressed to Sarah Rochel-Landau, Department of Pharmacology, Case Western Reserve University, School of Medicine, Cleveland, $\mathrm{OH} 44106$.

Copyright (C) 1988 Society for Neuroscience $0270-6474 / 88 / 010332-07 \$ 02.00 / 0$
}

achieved. On the other hand, the transient increase of transmitter release in nondenervated terminals may reflect either increased axonal transport of synaptic components during the sprouting period or greater excitatory drive.

Partial denervation of mammalian muscle is generally followed by sprouting of the remaining intact neurons that provide reinnervation of the original end-plate sites (Brown and Ironton, 1978; Slack and Hopkins, 1982). This process is associated with an increase of twitch tension generated by the intact axons (Edds, 1953; Guth, 1962). However, it is unclear whether motor neurons forced to innervate 3-5 times their original field maintain a normally high safety factor of transmitter release at all synapses within the expanded motor unit or whether the safety factor is reduced. Indeed, there appears to be an upper limit to the functional expansion of the motor unit. For instance, the success of sprouting and synapse regeneration appears to depend on motor unit size. Thus, the peroneus tertius muscle, which has 11 motor units and 300 muscle fibers, exhibits better recovery than soleus muscle with 20-25 motor neurons supplying about 1500 fibers. Also, Gorio et al. (1983) found that functional collateral reinnervation in soleus muscle was reduced in proportion to the number of remaining motor units, when more than $75 \%$ of the units were axotomized. Thus, in this muscle, motor unit may expand only 3- to 5-fold (Thompson and Jansen, 1977; Brown and Ironton, 1978; Brown et al., 1981), possibly because of an upper limit on total nerve terminal surface or volume or quantal output. Indeed, a study in young growing mice (Slack and Hopkins, 1982) concluded that while control quantal content increased 4-5 times in 4 months, quantal content in sprouting nerves increased far less. This result supports the hypothesis that mature nerve terminals supplied by motor neurons with 3-5 times the normal terminal field will show a decrease in quantal content. Alternatively, the result may reflect only the smaller sprouting capacity of developing neurons as compared with mature adult neurons.

A second question regarding sprouting after partial denervation is whether it leads to a redistribution of the capacity to release transmitter, such that all terminals would generate similarly reduced quantal content (as reported in immature mice: Slack and Hopkins, 1982) or whether 2 classes of terminals with different quantal content would emerge: the original nondenervated junctions with maintained quantal content and the newly sprouted terminals with low quantal content. The latter alternative would be expected if postmaturational cytoskeletal stability preserved a normal delivery of synaptic components to established synapses, whereas newly sprouted synapses could be more limited in this respect. 


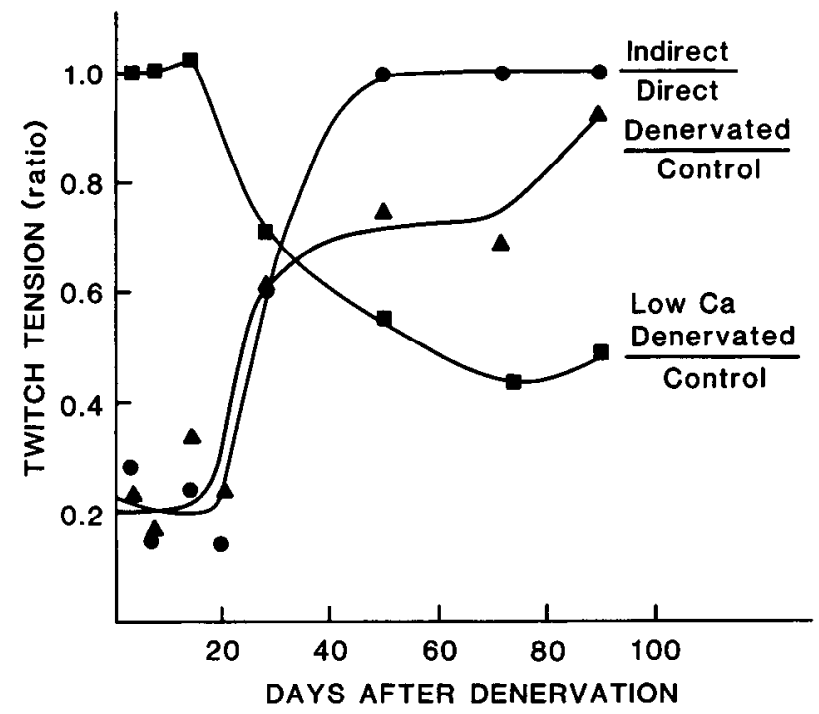

Figure 1. Recovery of twitch tension as a function of time after partial denervation. Filled circles, ratio of indirect to direct twitch in partially denervated muscles; filled triangles, ratio of indirect twitch of partially denervated muscle to that of contralateral control muscle; filled squares, ratio of indirect twitch in low $\mathrm{Ca} / \mathrm{high} \mathrm{Mg}$ solutions (partially denervated)/(contralateral control). Values at $28 \mathrm{~d}$ and later are means from 4-5 animals, and for these data, SE (not shown) were smaller than $10 \%$ of the mean. Values at 2-20 d are from 1-3 animals.

These 2 questions were addressed in this study by generating a 5-fold increase of mouse soleus motor unit size through partial denervation and by characterizing safety factor, transmitter release, and morphology in the resulting population of intact and newly sprouted nerve terminals.

\section{Materials and Methods}

Animals. Male CBF-1 mice (F-1 hybrid of Balb/c and C-57 strains, Charles River Laboratory) 6 to 7 months old and weighing 35-40 gm at the time of nerve section were used.

Nerve section. With the animal under pentobarbital anesthesia (60 $\mathrm{mg} / \mathrm{kg}$ ), the right L-5 nerve root was sectioned as previously described (Tiedt et al., 1977; Brown and Ironton, 1978). In order to prevent regeneration of the sectioned nerve, a $2 \mathrm{~mm}$ segment was removed, and the proximal nerve stump was inserted into the surrounding muscles.

Twitch tension. The efficacy of the L-5 section was evaluated by measurement of the twitch tension produced by indirect stimulation of the soleus motor nerve 2-3 d after denervation. Soleus muscles, together with a $1 \mathrm{~cm}$ length of motor nerve, were removed from mice under methoxyfluorane (Metofan. Pitman-Moore) anesthesia at indicated times after nerve section. The soleus muscles were placed in oxygenated Krebs solution at $30^{\circ} \mathrm{C}$, and the nerve was stimulated through a suction electrode at 3 times twitch threshold for $50 \mu \mathrm{sec}$. For direct muscle stimulation, the bathing fluid was withdrawn and the muscle stimulated by 2 silver wires straddling the central third of the muscle for $200 \mu \mathrm{sec}$. The twitch tension was measured with a Gould transducer and was recorded on a Brush pen-writer recorder with sufficient frequency response to reproduce the full twitch seen in parallel on a Tektronix oscilloscope. Twitch tension measurements provided the following parameters: (1) reduction of muscle twitch due to L-5 nerve section, compared with the contralateral side or compared with the ipsilateral direct twitch tension; (2) the number of remaining motor units (obtained by graded stimulation of the soleus nerve and counts of the number of increments in twitch size; see Brown and Ironton, 1978, and Grinnell and Trussel, 1983), and (3) a semiquantitative assay of the safety factor of transmitter release obtained by measuring the percentage twitch depression in low $\mathrm{Ca}(0.5 \mathrm{~mm}) / \mathrm{high} \mathrm{Mg}(1.9 \mathrm{~mm})$ solutions compared with the twitch in normal Krebs solution ( $2 \mathrm{~mm} \mathrm{Ca,} 1 \mathrm{~mm} \mathrm{Mg} \mathrm{Krebs).}$ In all experiments, the unoperated left soleus muscle was used as an internal paired control to minimize interanimal variability.

Intracellular recording. Electrophysiology was performed in vitro, as

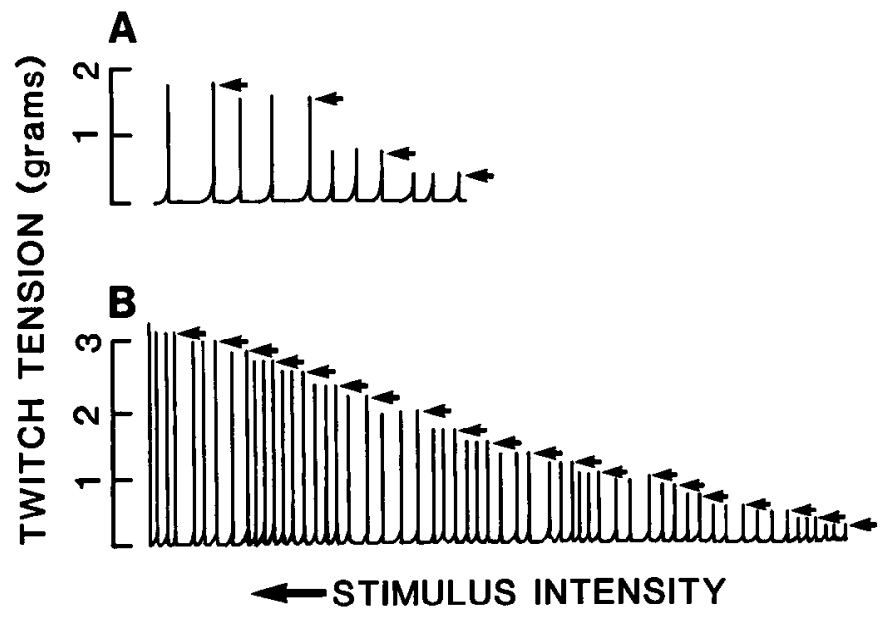

Figure 2. Estimate of the number of motor units per muscle by twitch tension increments. Each increment of twitch tension (as stimulus intensity was increased) represents the addition of 1 motor unit (arrow). $A$, Partially denervated muscle has 4 motor units. $B$, Control contains 21 motor units.

previously described (Robbins, 1981), using a suction electrode for nerve stimulation and glass microelectrodes $(10-20 \mathrm{M} \Omega$ resistance) to record miniature end-plate potentials (MEPPs) and end-plate potentials (EPPs). All data were digitized, stored, corrected to a resting potential of -80 $\mathrm{mV}$, and used for computation on a Northstar Horizon computer, as described previously (Kelly and Robbins, 1984). The Krebs solution was modified to contain $0.4 \mathrm{~mm} \mathrm{Ca}$ and $2.75 \mathrm{mM} \mathrm{Mg}$ and was maintained at $30^{\circ} \mathrm{C}$. Quantal content of evoked $\mathrm{ACh}$ release was calculated from the ratio of mean EPP amplitude to mean MEPP amplitude (direct quantal content) and, for confirmation, by the "method of failures," where quantal content $=\ln$ (number of events/number of failures) (Del Castillo and Katz, 1954). Only data in which both methods gave comparable results were included. For each muscle fiber, average MEPP amplitude and frequency were derived from recording 50 MEPPs, and the EPP delay, amplitude, and number of failures were averaged from a train of 100 EPPs at $2 \mathrm{~Hz}$ (after omitting the first 10 EPPs of the tetanus).

Histology. Nerve terminals of soleus muscles were stained with zinc iodide-osmium by the method of Akert and Sandri (1968). Briefly, the muscles were incubated overnight in a solution of zinc iodide- $0.5 \%$ osmium in the dark, then washed 3-4 times in distilled water, and stored in $0.9 \% \mathrm{NaCl}$ containing $0.02 \% \mathrm{Na}$-azide. Groups of $2-5$ muscle fibers were then teased apart and mounted on slides in glycerogel for microscopic observation. The fibers were inspected in the light microscope with a $100 \times$ Neofluor oil-immersion objective, and terminals were drawn by means of a camera lucida. Fiber diameter, nerve terminal area, number of terminal "regions," perimeter, and total length of terminal, as well as the presence of myelinated axons were evaluated. "Regions" are defined as areas of the nerve terminal that are separated from one another by thin or nonvisualized axonal connections (for example, in Fig. 6 there are 4 separate regions). "Terminal area" is defined as the total 2-dimensional area occupied by the terminal regions (for additional details of analysis, see Robbins and Fahim, 1985). The existence of myelinated axons in these preparations is of interest only when present. Negative findings could result from capricious staining, the loss of smaller (myelinated) axons when the fibers were teased apart, or the difficulty of distinguishing small lightly myelinated preterminal axons.

Student's $t$ test was used in all statistical comparisons between groups (or subgroups), and a level $<0.05$ was considered significant.

\section{Results}

Muscle twitch tension following partial denervation

Section of the L-5 spinal root reduced total twitch tension to a fraction of the original value 2-3 d after nerve section (Fig. 1). At that time, the ratio of indirect to the direct twitch was $20 \%$ of control, as was the ratio of indirect twitch of partially de- 

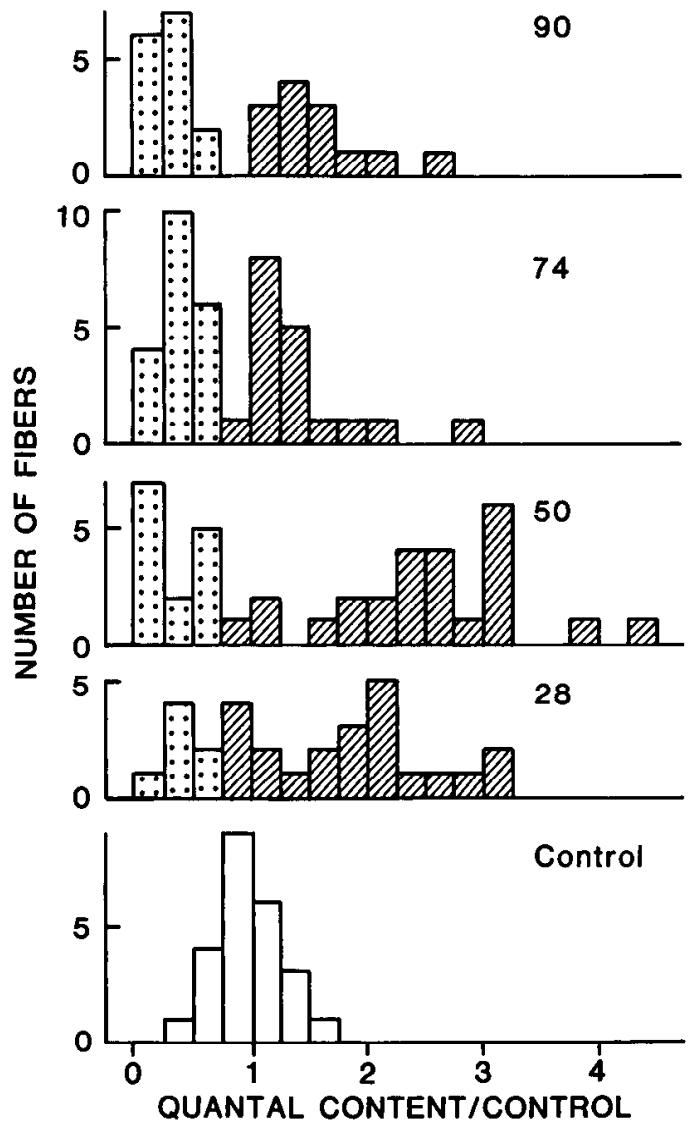

Figure 3. Histogram of quantal content of evoked ACh release 28, 50, 74 , and $90 \mathrm{~d}$ after L-5 root nerve section. Data from each animal are expressed as ratio of quantal content to the mean of contralateral control. Open bars, Control; dotted bars, group with "small" quantal content; cross-hatched bars, group with large quantal content (see text for classification scheme). The same classification into groups with "small" or "large" quantal contents is used in Figures 4 and 5.

nervated to control muscles. Thus, about $20 \%$ of the soleus innervation remained available to sprout to the remaining $80 \%$ of the muscle. The number of motor units remaining after L-5 section was estimated from the number of increments in twitch tension produced by graded increase of stimulus strength to the motor nerve (Fig. 2).

Two to seven motor units (average, 4.5) were detected 2-3 d after L-5 scction, whilc the control contained a mean of 21 motor units (range, 18-24). Similar observations were made by others (Tiedt et al., 1977; Brown and Ironton, 1978; Slack and Hopkins, 1982). Also 2-3 d after nerve section, there was no change in the depression of twitch tension by low $\mathrm{Ca} / \mathrm{high} \mathrm{Mg}$ solutions compared with controls (Fig. 1). This indicated that transmitter release of the remaining motor units was unaltered at this time.

The indirect twitch recovered slowly, reaching a maximum between 28 and $50 \mathrm{~d}$ after L-5 section (Fig. 1). The average number of remaining units did not increase from 28 to $90 \mathrm{~d}$ after nerve section, indicating that twitch recovery after $20 \mathrm{~d}$ was due to sprouting of existing units, and not to reinnervation by L-5 axons. However, even after the sprouted motor units achieved full (indirect/direct) twitch tension at 50-90 d, the twitch response in low $\mathrm{Ca} /$ high $\mathrm{Mg}$ solution was 2-3 times less than in the control muscle (Fig. 1). Thus, the safety factor of at lcast part of the sprouted motor units remained reduced. An

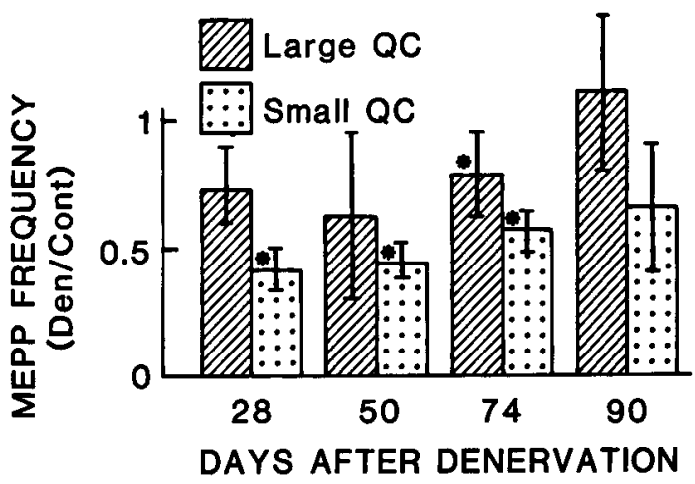

Figure 4. MEPP frequency as function of quantal release after partial denervation. Data are expressed as the mean ratio of MEPP frequency of partially denervated to control fibers. Large and small quantal contents were grouped as in Figure 3. The asterisks $\left(^{*}\right)$ indicate significant differences from contralateral control $(p<0.05)$. Vertical lines indicate SD based on 4-5 muscles at each time point, and 15-25 fibers per experimental group. Mean MEPP frequency in control muscles was 0.95 Hz.

intracellular quantal analysis (see below) was undertaken to elucidate the basis for this reduction. The twitch response in low $\mathrm{Ca} /$ high $\mathrm{Mg}$ solution also served to establish that the safety factor of the L4 units remaining $5 \mathrm{~d}$ after elimination of L5 axons was not different from that of the combined L4 and (majority) L5 units in unoperated muscles. Therefore, changes in transmitter release of $\mathrm{L} 4$ units at later times after denervation (see below) cannot be attributed to initially different levels of transmitter release from L4 and L5 units.

\section{Intracellular recording}

\section{Quantal content}

Quantal content of fibers in partially denervated and contralateral muscles was analyzed at various times after L-5 root nerve section (Fig. 3). Values were normalized to those of the paired contralatral control muscle (absolute value of control quantal content was $1.18 \pm 0.3$ ). From 28 to $90 \mathrm{~d}$ after L-5 section, a bimodal distribution of quantal content was evident. In order to standardize the separation of data into 2 modes, values of quantal content with the normalized ratios (Fig. 3) $\leq 0.75$ were placed in the "smaller mode," and the remainder of values were classified as the "larger mode." For instance, at $28 \mathrm{~d}$ (Fig. 3), the mean of the smaller mode was 0.5 and that of the larger was 2 . The mean of the smaller mode remained practically unchanged from 28 to $90 \mathrm{~d}$ after operation, while the mean of the larger mode, which was twice the control value at $50 \mathrm{~d}$, decreased thereafter to a value of about 1.4 times control at 74 and $90 \mathrm{~d}$ (Fig. 3). While the changes at 28 and $50 \mathrm{~d}$ were clearly different from control, those at 74 and $90 \mathrm{~d}$ were only slightly larger than control, and were similar.

All data showing deviations of direct quantal content from control values mainly reflected changes in EPP rather than in MEPP amplitudes. MEPP amplitudes were not more than $25 \%$ different from control in any experimental group, and EPP amplitudes changed in proportion to indirectly calculated quantal content, e.g., from $0.635 \mathrm{mV}$ control to either $0.257 \mathrm{mV}$ (smaller mode) or $0.825 \mathrm{mV}$ (larger mode) at $28 \mathrm{~d}$ after partial denervation.

It is likely that the nondenervated terminals remaining after partial denervation were those with normal or increased quantal content, while the newly formed terminals had a reduced quantal 


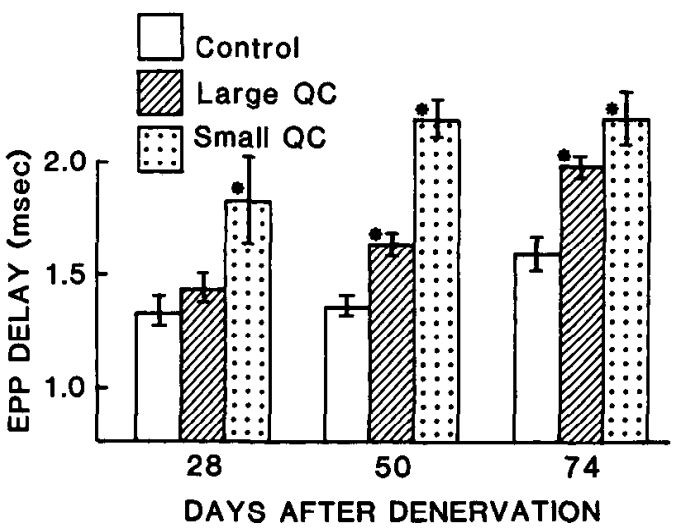

Figure 5. EPP latency as a function of quantal content, with fibers grouped as in Figures 3 and 4 and presented as a function of time after partial denervation. The asterisks $\left(^{*}\right)$ indicate significant differences from control $(p<0.05)$. Vertical lines are SD. Data for each experimental bar were derived from 15-25 fibers in 4-5 muscles.

content. This hypothesis was tested by determining whether terminals with high and low quantal contents showed other physiological properties (MEPP frequency and conduction delay) typical of established or newly developed terminals, respectively.

\section{MEPP frequency}

Since low MEPP frequency is characteristic of newly regenerating nerve terminals (Tonge, 1974; Carmignoto et al., 1983), we sought correlations between MEPP frequency and quantal content of the same terminals. In contralateral control muscle fibers, the absolute value of quantal content was about 1.2 and MEPP frequency was about $1 \mathrm{~Hz}$. The frequencies were only $20 \%$ less in control fibers with low quantal content $(<0.75$ of the mean). In partially denervated muscles, MEPP frequency of the large mode quantal content group was not significantly different from control (with one exception at $74 \mathrm{~d}$ ), but in the group with quantal contents $<0.5$, MEPP frequency was $<0.5 \mathrm{~Hz}$ (Fig. 4). At $74 \mathrm{~d}$ after operation, mean MEPP frequency of fibers with larger quantal content was lower than control, probably because some sprouted terminals were now releasing sufficient transmitter to enter the larger mode classification, even though MEPP frequency was still depressed. The correlation between low quantal content and low MEPP frequency indicates that this group of muscle fibers was mainly supplied by sprouted nerve terminals.

\section{EPP latency}

Nerve conduction is characteristically slower at newly regenerated neuromuscular junctions (Miledi, 1960; Bennett et al., 1973; Dennis and Miledi, 1974). Therefore, the latency from nerve stimulation to the foot of the EPP was evaluated as a function of quantal content (Fig. 5). In the control group, quantal content was 1.2 and EPP latency $1.3 \mathrm{msec}$, and the same latency was found in control fibers with lower quantal content $(<0.75$ of the mean).

In the partially denervated muscles, the group with larger quantal content showed a conduction delay of $1.5 \mathrm{msec}$, slightly longer than control (probably because of the inclusion of a few sprouted fibers, especially at 50 and $74 \mathrm{~d})$. However, in the group with smaller quantal content $(0.4)$, the mean latency (of
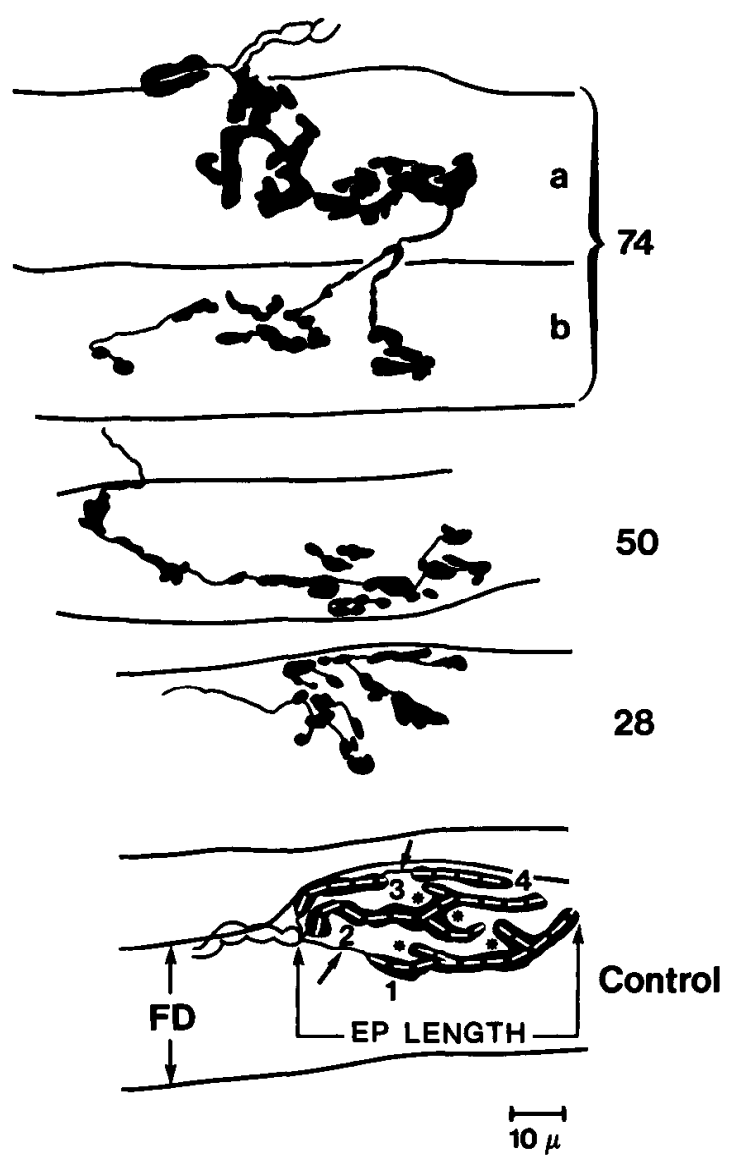

Figure 6. Camera lucida drawings of nerve terminals stained with zinc iodide-osmium. Days after partial denervation are indicated at the right of each drawing. Scale bar, $10 \mu \mathrm{m}$. In bottom tracing, the asterisk $\left(^{*}\right)$ indicates branch point, numbers $1-4$ indicate "regions," arrows indicate connection between regions; $F D$, fiber diameters; $E P$, end plate. Dashed lines indicate midline length of terminals. Area is the area included within the perimeter of the black terminal regions.

about 2 msec) was invariably greater than control. This result provided still further evidence that terminals with low quantal content were mainly newly sprouted terminals, while those with large quantal content were mainly intact.

\section{Nerve terminal morphology}

Morphometric measurements of zinc iodide-osmium-stained terminals (parameters illustrated in Fig. 6) were evaluated to determine whether original and reinnervated terminals were distinguishable on the basis of nerve terminal area, number of discrete "regions" (Robbins and Fahim, 1985), and presence or absence of a preterminal myelinated axon (see Materials and Methods for definitions and qualifications). Nerve terminal area was chosen as a significant parameter because it is correlated with end-plate size and quantal release (Kuno et al., 1971). The number of discrete terminal regions was chosen as a second criterion to distinguish sprouted from intact terminals since an increased number of regions is characteristic of newly regenerated mammalian nerve terminals (N. Robbins, unpublished observations). Finally, the presence of nerve terminals supplied by myelinated axons (Table 1) was a further indicator of intact terminals. However, in teased preparations, the incoming axon or sprout was often missing, and lightly myelinated or unmyelinated axons might be more readily detached during dissection, 
Figure 7. Histograms of nerve terminal morphological parameters. $A$ and $C$, Control; $B$ and $D, 28 \mathrm{~d}$ partially denervated muscle. $A$ and $B$ are nerve terminal area; $C$ and $D$, the number of nerve terminal regions. Arrows indicate the mean area of control or large and small terminals. Dotted boxes are fibers with areas $<250 \mu \mathrm{m}^{2} ;$ cross-hatched boxes are control fibers or fibers with areas $>250 \mu \mathrm{m}^{2}$

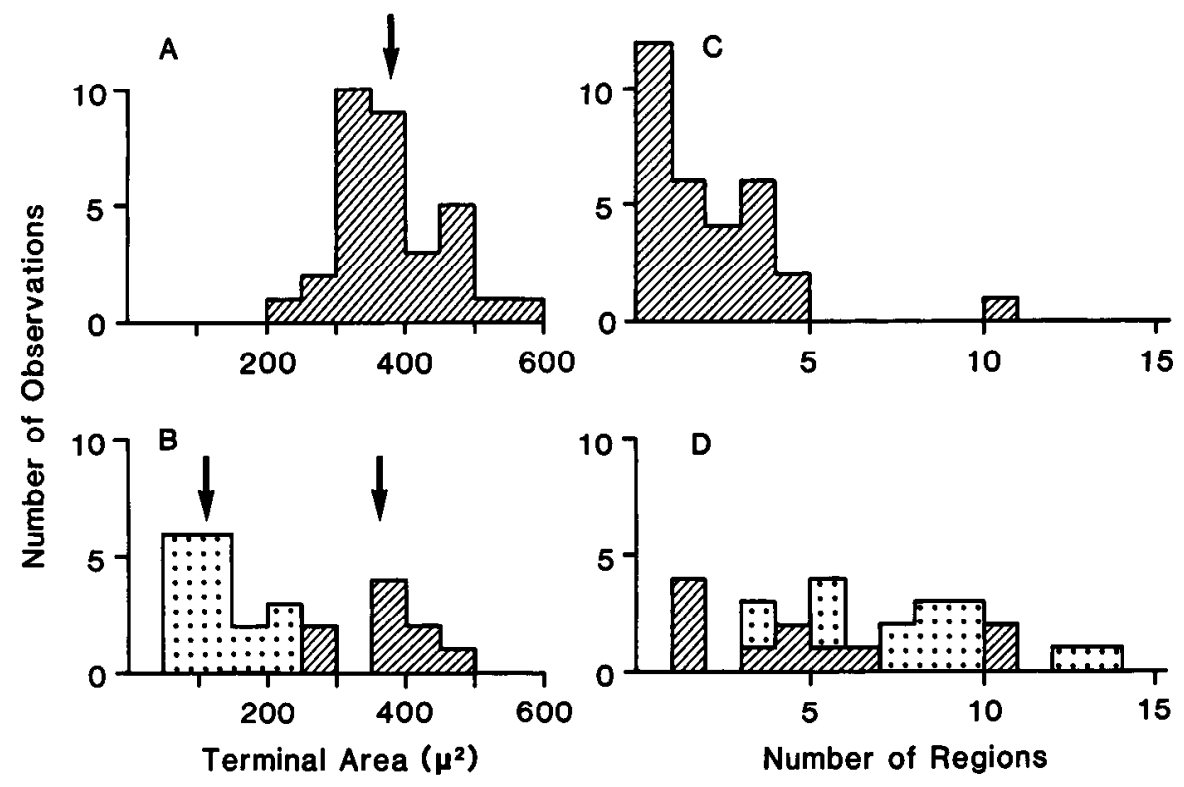

so that this indicator was supportive but not absolute. Indeed, other methods recently applied to partially denervated preparations (J. Jacob and N. Robbins, unpublished observations) have revealed that by $60 \mathrm{~d}$, almost all nerve terminals become partially myelinated.

In control terminals (e.g., Fig. 6, bottom tracing), nerve terminal area was typically $300-500 \mu \mathrm{m}^{2}(10 \times 30 \mu \mathrm{m}$; Fig. $7 A)$, with scveral terminal branches, 1-5 separate terminal regions (Fig. $7 C$, Table 1), and frequent presence of a myelinated preterminal axon (Table 1). In partially denervated muscle, the

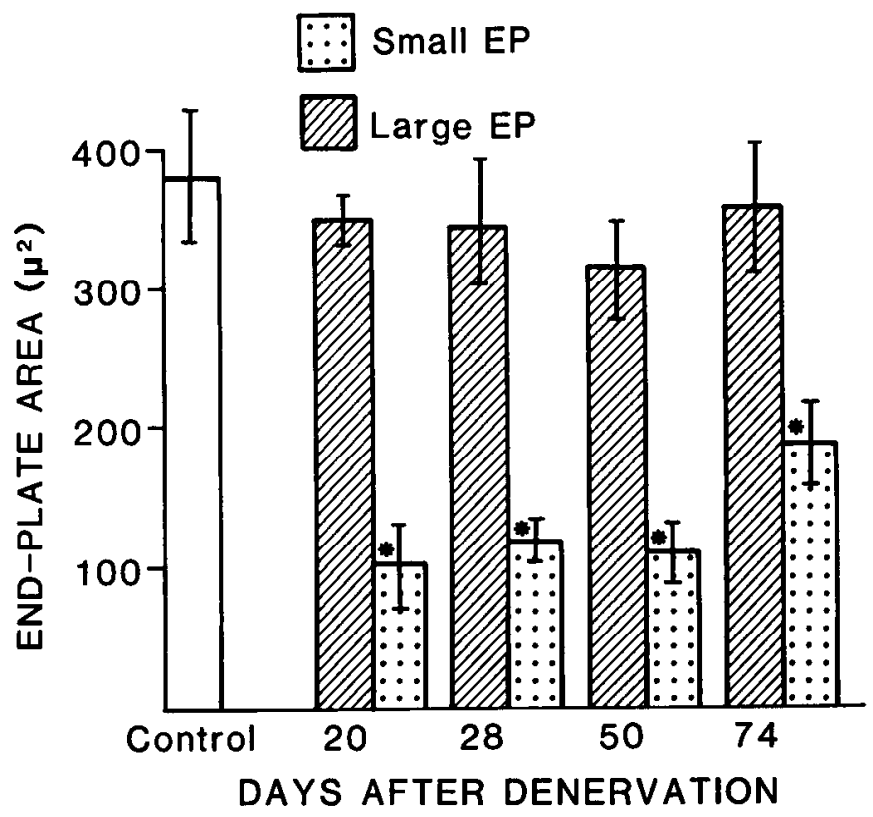

Figure 8. Nerve terminal area after partial denervation. In partially denervated muscle, all bar heights are mean nerve terminal areas of "small" (dotted bars) or "large" (cross-hatched bars) nerve terminals, depending on whether the terminal area was less than or greater than $250 \mu \mathrm{m}^{2}$, respectively. Asterisks $\left(^{*}\right)$ indicate significant differences from control $(p<0.05)$. Vertical lines indicate SD. Each data point is the mean of 12-19 fibers taken from 4-5 muscles, except for control values (32 fibers) and the $20 \mathrm{~d}$ large group ( 3 fibers). population of terminals could be divided into 2 groups (e.g., Fig. $7 B)$ : those with nerve terminal area $<250 \mu \mathrm{m}^{2}(<86 \%$ of control sample) and those with greater values. The "large" group was similar to controls in appearance, number of regions, and presence of myelinated preterminal axons (Figs. 7, $B, D ; 8$; Table 1). However, the presence of terminal sprouts increased the longitudinal end-plate length ("EP length" in Fig. 6) by $44 \%$ at $20 \mathrm{~d}$ or more after partial denervation. This increase in nerve terminal length was brought about by a few small boutons made by sprouts leaving the end plates along their path to the new muscle. Contacts of this type did not significantly increase the area of the terminal, although the end-plate "length" (the longitudinal distance from the first to the last contact region of the end plate) was thereby increased. It is interesting to note that there was no increase of terminal area in the "largc" group (Fig. 8 ), despite the increased quantal release of the intact terminals. The second type of terminal, with relatively small area (Figs. 6, $7 B, 8$ ), was less branched and contained a large number (6-20) of separate terminal regions (Figs. 6, 7D; Table 1). These terminals were supplied by thin unmyelinated nerve sprouts that arose from neighboring normal terminals (where tracing was possible) and were never associated with myelinated axons (Table 1). These are all expected characteristics of sprouted terminals. Indeed, the presence of small terminals even at $74 \mathrm{~d}$, when recovery of the single twitch was almost complete, appears to account for the class of fibers with reduced quantal content and low MEPP frequency, while the thin nerve sprout supplying these terminals would have led to a relatively longer EPP latency. The end-plate physiology and morphometry were studied in the same muscles, but without one-to-one correlation. Thus, there is only an indirect correlation between the physiological and the morphological evidence for reduction of ACh release in sprouting terminals.

\section{Discussion}

As described in the introduction, 2 questions motivated this study. The first was whether motor neurons forced to innervate 3-5 times the normal number of motor units would be able to maintain a normal safety factor of transmitter release in all fibers of the expanded motor unit. The studies of twitch tension in 
Table 1. Myelination and number of regions per nerve terminal in control, "large," and "small" terminals ${ }^{a}$

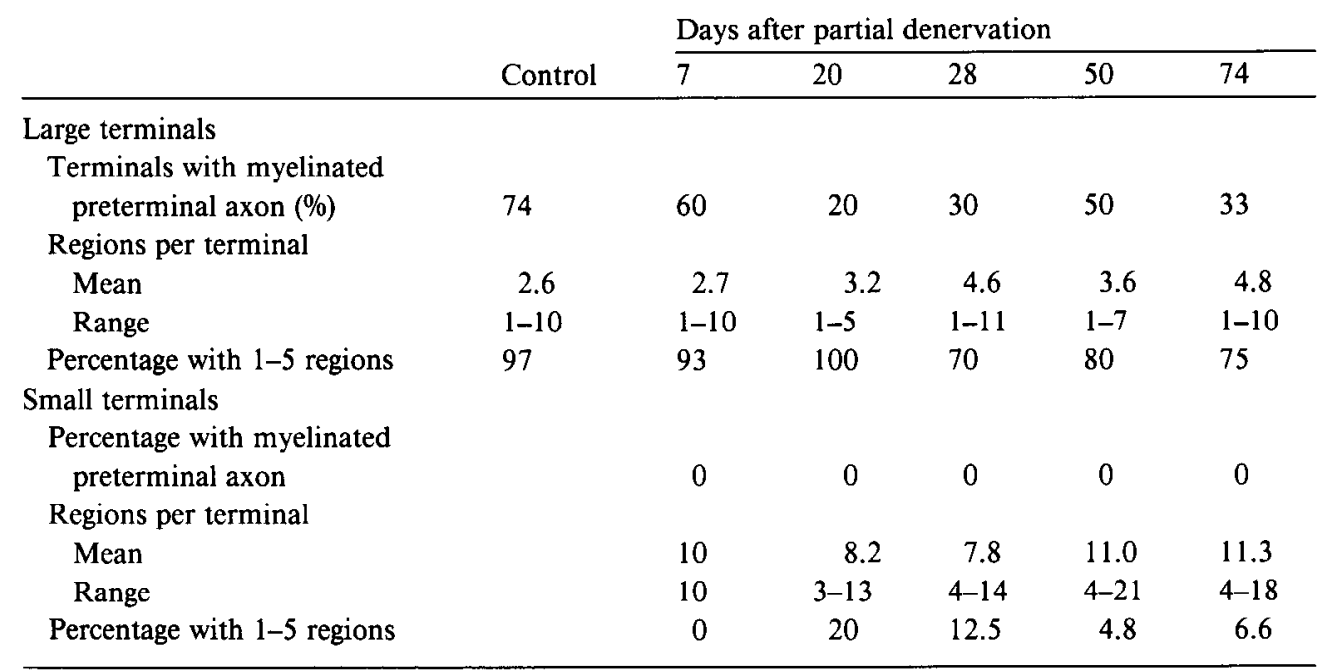

"In partially denervated muscles, terminals $<250 \mu \mathrm{m}^{2}$ in area were classified as "small," and the remainder, which overlapped control values, were considered "large." Each value is the mean of 12-19 fibers taken from 4-5 muscles, except for control values ( 32 fibers) and the $20 \mathrm{~d}$ large group ( 3 fibers).

normal and in low $\mathrm{Ca} /$ high $\mathrm{Mg}$ solutions clearly indicated that although normal twitch tension was restored by $50 \mathrm{~d}$ after operation, the safety factor was reduced for at least $90 \mathrm{~d}$.

A second question was whether partial denervation and collateral innervation would lead to a redistribution of the capacity to release transmitter such that all junctions would have similarly reduced quantal content or whether 2 classes of fibers with different quantal content would emerge-the original nondenervated junctions with maintained quantal content and the newly sprouted junctions with low quantal content. A parallel question was posed with respect to redistribution of nerve terminal area. In fact, 2 populations of junctions were found from 28 to $90 \mathrm{~d}$ after partial denervation. One population, with normal or greater than normal quantal content, almost certainly consisted of the original terminals with intact innervation (and possibly some well-established sprouted terminals). This was indicated by the normal impulse latency, MEPP frequency, nerve terminal area, number of terminal regions, and presence (where included in the specimen) of innervation by a myelinated axon. On the other hand, the population of nerve terminals with relatively low quantal content consisted of newly sprouted terminals, as indicated by increased latency, low MEPP frequency (Tonge, 1974; Carmignoto et al., 1983), small terminal area, large number of regions, and innervation (where visualized) by thin unmyelinated sprouts. Our results differ from those of Slack and Hopkins (1982), who found only a unimodal population of muscle fibers with reduced quantal contents after partial denervation in 20 - to 30 -d-old mice. Since at that age, quantal content was 8 times less than that of mature animals (see Fig. 2 in Slack and Hopkins, 1982), whereas 5- to 7-month-old, mature mice were used in the present study, the presence of growing nerve terminals may have affected the response to partial denervation. Alternatively, the difference in outcomes may depend on the fact that in soleus, used by us, most collateral sprouts arise from nerve terminals (Edds, 1953), whereas in the tensor fascia lata muscle used by Hopkins and Slack (1981), collateral sprouts originate from intramuscular nodes of Ranvier.
An important observation was that expansion of the motor neuron terminal field led to reduced nerve terminal size and decreased quantal content in the newly added junctions (see also Hopkins and Slack, 1981). The implication is that there is an upper limit to the amount of transmitter released per motor neuron. In the present study, the motor unit size was cxpanded about 4-fold, and the quantal content of presumably sprouted terminals was about half of normal; therefore, total output per remaining neuron was about 2.5 times normal ( 1 part normal, 3 parts $1 / 2$ normal). Interestingly, total nerve terminal area of the motor neuron would also have expanded to about the same extent (2.5-fold), suggesting that an upper limit to the supply of membrane or terminal components restricts expansion of both nerve terminal size and quantal output. This result complements the reported increase in quantal content when the motor neuron terminal field is experimentally reduced (Grinnel and Herrera, 1981). An alternative interpretation is that the newly formed terminals may exhibit less release not because of some upper limit to the total terminal size, but because at 60-90 d the new functionally effective terminals receive feedback that arrests further growth. However, in other studies of denervation (see in introduction), in which a larger percentage of innervation was preserved, the innervation process apparently proceeded to complete restoration of the original safety factor. Thus, when terminal load is reduced, the system achieves full transmitter release in sprouted fibers.

From a functional point of view, the neuron in a partially denervated muscle "spreads" its available transmitter release capacity among the sprouted recipient fibers such that no fibers are below threshold under normal circumstances, even though the safety factor is reduced by a factor of 2 . However, the sprouted junctions might be compromised under heavy tetanic stress, when rundown reduces EPP amplitude by about two-thirds (Robbins and Fischbach, 1971). Spreading of quantal content among the sprouted terminals would occur if those muscles that were initially reinnervated by terminals releasing subthreshold amounts of ACh per impulse released sprouting factors (e.g., Gurney, 1984) that enhanced terminal growth, causing a rcdis- 
tribution of available transmitter machinery among the immature sprouting terminals.

An unexpected finding was that the intact terminals of the remaining motor units exhibited a transient increase of quantal content. This (and preliminary reports by Grinell, 1985, and Rochel and Robbins, 1985) is the first report of transient increases of quantal content following partial denervation, although an increase in muscles contralateral to totally denervated muscles has been observed (Herrera and Grinnell, 1980). Since the area of the presumed intact terminals in partially denervated muscles was not greater than control, a morphologic or physiological mechanism other than simple expansion of synaptic terminal must be considered to account for the increase in quantal release. Increase of synaptic contact area or of number of active zones (e.g., Herrera et al., 1985) or altered terminal ion channel density might be possible mechanisms.

The enhanced transmitter release of the intact motor unit may have resulted from a greater excitatory drive after partial denervation. An alternative interpretation is that the regenerative response after partial denervation, like that after axotomy, increases neuronal biosynthesis of components necessary to sustain sprouting (McQuarrie, 1984; Willard et al., 1984). Indeed, it was reported that partial denervation acted as a "conditioning lesion" which increased the rate of axonal regeneration after a second (axotomy) lesion 2 weeks later (Sparrow and Grafstein, 1983). Therefore, increased fast axonal transport of synaptic membrane components in response to nerve terminal sprouting may give rise to the transient increase in quantal release during the period when new terminals are actively being formed. This will become a testable hypothesis as the transported molecular components underlying synaptic transmission become better defincd.

\section{References}

Akert, K., and C. Sandri (1968) An electron-microscopic study of zinc iodide-osmium impregnation of neurons. I. Staining of synaptic vesicles at cholinergic junctions. Brain Res. 7: 286-295.

Bennett, M. R., E. M. McLachlan, and R. S. Taylor (1973) The formation of synapses in reinnervated mammalian striated muscle. J. Physiol. (Lond.) 233: 481-500.

Brown, M. C., and R. Ironton (1978) Sprouting and regression of neuromuscular synapses in partially denervated mammalian muscles. J. Physiol. (Lond.) 278: 325-348.

Brown, M. C., R. L. Holland, and W. G. Hopkins (1981) Motor nerve sprouting. Annu. Rev. Neurosci. 4: 17-42.

Carmignoto, G., M. Finesso, R. Siliprandi, and A. Gorio (1983) Muscle reinnervation. I. Restoration of transmitter release mechanisms. Neuroscience 8: 393-401.

Del Castillo, M., and B. Katz (1954) Quantal components of endplate potential. J. Physiol. (Lond.) 124: 560-573.

Dennis, M. J., and R. Miledi (1974) Characteristics of transmitter release at regenerating frog neuromuscular junctions. J. Physiol. (Lond.) 239: 571-591.

Edds, M. V., Jr. (1953) Collateral nerve regeneration. Q. Rev. Biol. 28: $260-276$.

Gorio, A., P. Marini, and R. Zanoni (1983) Muscle rcinncrvationIII. Motoneuron sprouting capacity, enhancement by exogenous gangliosides. Neuroscience 8: 417-429.

Grinnell, A. (1985) Partial denervation of the frog sartorius causes enhanced transmitter release of surviving junctions. Soc. Neurosci. Abstr. 11: 916.

Grinnell, A. D., and A. A. Herrera (1981) Specificity and plasticity of neuromuscular connections: Long-term regulation of motor neuron function. Prog. Neurobiol. 17: 203-282.

Grinnell, A. D., and L. O. Trussell (1983) Synaptic strength as function of motor unit size in the normal frog sartorius. J. Physiol. (Lond.) 338: 221-241.

Gurney, M. E. (1984) Suppression of sprouting at the neuromuscular junction by immune sera. Nature $307: 546-548$.

Guth, L. (1962) Neuromuscular function after regeneration of interrupted nerve fibers into partially denervated muscle. Exp. Neurol. 6 : 129-141.

Herrera, A. A., and A. D. Grinnell (1980) Transmitter release from frog motor nerve terminals depends on motor unit size. Nature 287: 649-651.

Herrera, A. A., and A. D. Grinnell (1981) Contralateral denervation causes enhanced transmitter release from frog motor nerve terminals. Nature 291: 495-597.

Herrera, A. A., A. D. Grinnell, and B. Wolowske (1985) Ultrastructural correlates of experimentally altered transmitter release efficacy in frog motor nerve terminals. Neuroscience 16: 491-500.

Hopkins, W. G., and J. R. Slack (1981) The sequential development of nodal sprouts in mouse muscle in response to nerve degeneration. J. Neurocytol. 10: 537-556.

Kelly, S. S., and N. Robbins (1984) Bimodal miniature and evoked end-plate potentials in adult mouse neuromuscular junctions. J. Physiol. (Lond.) 346: 353-363.

Kuno, M., S. A. Turkanis, and J. N. Weakly (1971) Nerve terminal size and transmitter release at the neuromuscular junction of the frog. J. Physiol. (Lond.) 213: 545-556.

McQuarrie, I. G. (1984) Effect of conditioning lesion on axonal transport during regeneration: The role of slow transport. In Axonal Transport in Neuronal Growth and Regeneration, J. S. Elam and P. Cancalon, eds., pp. 195-209, Plenum, New York.

Miledi, R. (1960) Properties of regenerating neuromuscular synapses in the frog. J. Physiol. (Lond.) 154: 190-205.

Robbins, N. (1981) Neurotrophic regulation of rat muscle glucose 6-phosphate dehydrogenase in vitro. Brain Res. 225: 387-399.

Robbins, N., and M. A. Fahim (1985) Progression of age changes in mature mouse motor nerve terminals and its relation to locomotor activity. J. Neurocytol. 14: 1019-1036.

Robbins, N., and G. D. Fischbach (1971) Effect of chronic disuse of rat soleus neuromuscular junction on presynaptic function. J. Neurophysiol. 34: 570-578.

Rochel, S., and N. Robbins (1985) Effect of expansion of motor neuron terminal field on acetylcholine release. Soc. Neurosci. Abstr. 11:916.

Slack, J. R., and W. G. Hopkins (1982) Neuromuscular transmission at terminals of sprouted mammalian motor neurons. Brain Res. 237: 121-135.

Sparrow, J. R., and B. Grafstein (1983) Prior collateral sprouting enhances axonal regeneration. Brain Res. 269: 133-136.

Thompson, W., and J. K. Jansen (1977) The extent of sprouting of remaining motor units in partly denervated immature and adult rat muscles. Neuroscience 2: 523-535.

Tiedt, T. N., E. X. Alburquerque, and L. Guth (1977) Degenerating nerve fiber products do not alter physiological properties of adjacent innervated skeletal muscle fibers. Science 198: 839-841.

Tonge, D. A. (1974) Physiological characteristics of reinnervation of skeletal muscles in the mouse. J. Physiol. (Lond.) 241: 141-153.

Willard, M., J. H. P. Skene, C. Simon, K. Meiri, N. Hirokawa, and M. Glicksman (1984) Regulation of axon growth and cytoskeletal development. In Axonal Transport in Neuronal Growth and Regeneration, J. S. Elam and P. Cancolon, cds., pp. 171-183, Plenum, Ncw York. 